\title{
Pleuroparenchymal fibroelastosis in patients with pulmonary disease secondary to bone marrow transplantation
}

\author{
Jan $\mathrm{H}$ von der Thüsen ${ }^{1}$, David $\mathrm{M} \mathrm{Hansell}^{2}$, Masaki Tominaga ${ }^{1,3}$, Paul A Veys ${ }^{4}$, \\ Michael T Ashworth ${ }^{5}$, Catherine M Owens ${ }^{6}$ and Andrew G Nicholson ${ }^{1}$ \\ ${ }^{1}$ Department of Histopathology, Royal Brompton and Harefield NHS Foundation Trust, London, UK; \\ ${ }^{2}$ Department of Radiology, Royal Brompton and Harefield NHS Foundation Trust, London, UK; ${ }^{3}$ Department \\ of Pathology, Toyama University Hospital, Toyama, Japan; ${ }^{4}$ Department of Bone Marrow Transplantation, \\ Great Ormond Street Hospital for Children NHS Trust, London, UK; ${ }^{5}$ Department of Paediatric \\ Histopathology, Great Ormond Street Hospital for Children NHS Trust, London, UK and ${ }^{6}$ Department of \\ Radiology, Great Ormond Street Hospital for Children NHS Trust, London, UK
}

\begin{abstract}
This study presents four patients who underwent bone marrow transplantation and subsequently developed pleuroparenchymal fibroelastosis, hitherto reported as an idiopathic condition. All presented clinically with pneumothorax and subpleural fibrosis on high-resolution computed tomography. In addition to the expected obliterative bronchiolitis, histopathology showed coexistent subpleural changes, and the relationship of pathology in multiple anatomic compartments in post bone marrow transplantation pulmonary disease is discussed. Modern Pathology (2011) 24, 1633-1639; doi:10.1038/modpathol.2011.114; published online 5 August 2011
\end{abstract}

Keywords: bone marrow transplantation; graft-vs-host disease; pleuroparenchymal fibroelastosis; pneumothorax

Bone marrow transplantation can lead to a variety of pulmonary complications, affecting $40-60 \%$ of patients, ${ }^{1,2}$ with a high rate of morbidity and mortality. ${ }^{1,3,4}$ Indeed, pulmonary complications are the commonest cause of death in bone marrow transplantation recipients. ${ }^{4}$ In the early phase, these are frequently of infectious aetiology, but late-phase complications (100 days or more after transplant) are predominantly non-infectious in origin, and often are related to graft-vs-host disease in allogeneic bone marrow transplantation. ${ }^{5-8}$ A common pattern of post bone marrow transplantation fibrosis is constrictive obliterative bronchiolitis, with airflow limitation and computed tomography (CT) changes of mosaic attenuation and air trapping. ${ }^{7-9}$ Its aetiology remains unresolved, with potential causes including viral infections, autoimmunity and graft-vs-host disease..$^{6,9,10}$

In the four cases described in the present study, we describe a pattern of pleuroparenchymal fibroelastosis

Correspondence: Dr JH von der Thüsen, PhD, MBBS, Department of Histopathology, Royal Brompton and Harefield NHS Foundation Trust, Sydney Street, London SW3 6NP, UK.

E-mail: j.vonderthuesen@rbht.nhs.uk

Received 7 January 2011; revised 1 June 2011; accepted 1 June 2011; published online 5 August 2011 following bone marrow transplantation, as diagnosed by CT findings and histopathology. Two of the cases were part of a previous CT report of complications post bone marrow transplantation. ${ }^{11}$ This histological pattern of interstitial fibrosis has hitherto only been reported in an idiopathic setting with the seven patients being adults (average age, 51 years) and six being female. ${ }^{12,13}$ As is the case in our patients, in those series, pleuroparenchymal fibroelastosis was found to predispose to secondary spontaneous or iatrogenic pneumothoraces. The first series included a patient with a sibling who had died of progressive pleuroparenchymal fibroelastosis, and two patients who had been treated with chemotherapy, but lacked a potentially unifying causal factor. We believe that we can now suggest a potential aetiology at least in a subgroup of patients with pleuroparenchymal fibroelastosis in that all of our patients had undergone bone marrow transplantation for a haematological disorder.

\section{Materials and methods}

Thin-section CT scans of four patients (two male, two female, age range: 13 to 55 years, average age: 
Table 1 Patient characteristics

\begin{tabular}{|c|c|c|c|c|c|}
\hline & & Case 1 & Case 2 & Case 3 & Case 4 \\
\hline & Sex & Female & Male & Male & Female \\
\hline & Underlying disease & Aplastic anaemia & $\begin{array}{l}\text { Acute myeloid } \\
\text { leukaemia }\end{array}$ & $\begin{array}{c}\text { Acute lymphoblastic } \\
\text { leukaemia }\end{array}$ & $\begin{array}{l}\text { Acute myeloid } \\
\text { leukaemia }\end{array}$ \\
\hline & Transplant type & $\begin{array}{l}\text { Allogeneic, } \\
\text { matched sibling }\end{array}$ & $\begin{array}{l}\text { Allogeneic, } \\
\text { sibling }\end{array}$ & Allogeneic, sibling & Autologous \\
\hline & $\begin{array}{l}\text { Time from bone marrow } \\
\text { transplantation to } \\
\text { presentation (in years) }\end{array}$ & 2 years 9 months & 12 & 16 & 2 \\
\hline & $\begin{array}{l}\text { Extra-pulmonary } \\
\text { graft-vs host disease }\end{array}$ & No & Yes & No & No \\
\hline \multirow[t]{3}{*}{$C T$} & & $\begin{array}{l}\text { Patchy visceral } \\
\text { pleural thickening }\end{array}$ & $\begin{array}{l}\text { Visceral pleural } \\
\text { thickening }\end{array}$ & $\begin{array}{l}\text { Non-uniform visceral } \\
\text { pleural thickening }\end{array}$ & $\begin{array}{c}\text { Pleuroparenchymal } \\
\text { irregularity consistent } \\
\text { with fibrosis }\end{array}$ \\
\hline & & Subpleural blebs & $\begin{array}{c}\text { Pleuroparenchymal } \\
\text { tags }\end{array}$ & Subpleural blebs & Fine interstitial fibrosis \\
\hline & & Mosaicism & Mosaicism & Mosaicism & Ground-glass opacification \\
\hline Pathology & $\begin{array}{l}\text { Method of } \\
\text { sampling }\end{array}$ & $\begin{array}{l}\text { Video-assisted } \\
\text { thoracoscopic } \\
\text { biopsy }\end{array}$ & Postmortem & $\begin{array}{l}\text { Video-assisted } \\
\text { thoracoscopic biopsy }\end{array}$ & Postmortem \\
\hline
\end{tabular}

31 years, median age: 28 years) who had undergone allogeneic bone marrow transplantation, all of whom subsequently developed late-onset pulmonary complications, including recurrent pneumothoraces, were analysed. Histological analysis was performed after routine Haematoxylin \& Eosin and Elastica-van Gieson staining of sections of formalin-fixed, paraffinembedded tissue (FFPE). Patients' characteristics are summarised in Table 1.

\section{Results}

\section{Case 1}

A 5-year-old daughter of non-consanguineous parents presented with recurrent viral infections, and was diagnosed with aplastic anaemia. At the age of 10 years, she received a cyclophosphamide-conditionedmatched sibling donor and saved cord blood bone marrow transplantation. Two years after bone marrow transplantation, she developed recurrent chest and shoulder pain, increasing lethargy and increasing breathlessness, with some biphasic wheezing and occasional fine crackles. A chest radiograph showed bilateral pneumothoraces.

On high-resolution CT, there were small bilateral pneumothoraces; in addition to some subpleural blebs and reticular elements at the apices (Figure 1a), there was conspicuous thickening of the visceral pleura, particularly over the upper lobes (Figures 1a and b). Elsewhere there was a mosaic attenuation pattern consistent with constrictive obliterative bronchiolitis.

Bilateral video-assisted thoracoscopic apical bullectomies were performed that showed marked, sharply demarcated established subpleural fibrosis with extensive elastin deposition. This was associated with mild patchy chronic inflammation and obliterative bronchiolitis. A year after the procedure, the patient has shown slight benefit from methylprednisolone therapy, with continued deterioration in lung function (FEV1 29\% predicted, FVC 31\% 

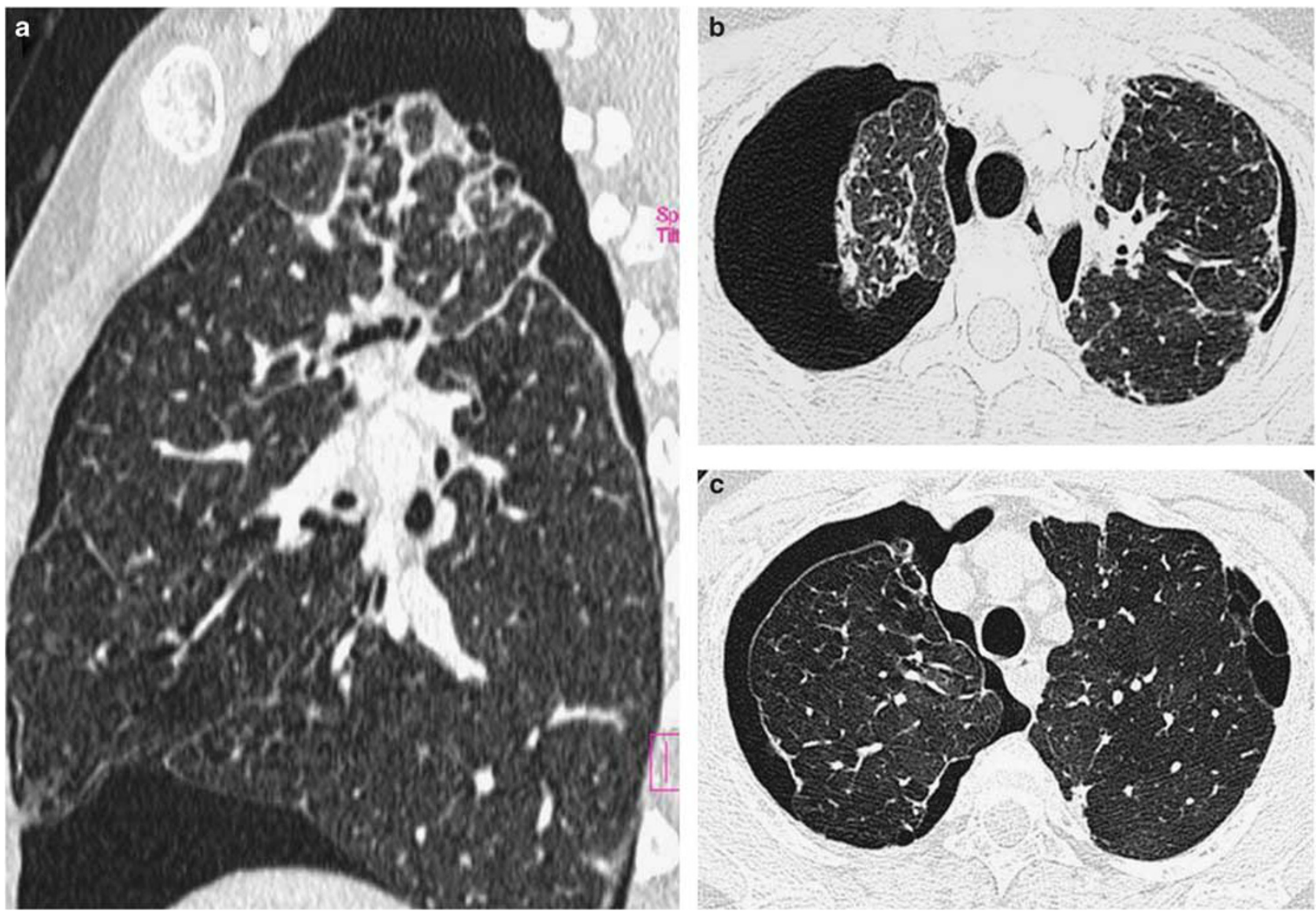

Figure 1 Case 1: (a) coronal re-format showing a right pneumothorax and the upper lobe predominance of the pleuroparenchymal disease. Note the thickening of the visceral pleural surface, particularly posteriorly, and the small subpleural blebs at the apex. (b) Transverse computed tomography (CT) demonstrating bilateral pneumothoraces and visceral pleural thickening. Case 3: (c) transverse CT showing small right pneumothorax (other sections revealed a minute left pneumothorax) and subpleural bleb at the left apex. There is patchy thickening of the visceral pleura around the right lung apex and subtle mosaic attenuation pattern in the left lung consistent with obliterative bronchiolitis.

predicted), requiring home oxygen overnight, and a poor prognosis.

\section{Case 2}

At the age of 11 years, this male patient was diagnosed with acute myeloid leukaemia. He received chemotherapy, followed by total body irradiation and a sibling bone marrow transplantation. He had mild graft-vs-host disease post transplant. He developed respiratory problems at the age of 17 years, which were believed to relate to obliterative bronchiolitis with mild left lower lobe bronchiectasis. Four years later, he was admitted with a right-sided pneumothorax, which recurred 2 months later, and was treated with talc pleurodesis. He was readmitted several times with progressive interstitial pulmonary fibrosis.

High-resolution CT scans showed some regional inhomogeneity, in the density of the lung parenchyma, consistent with the previously documented small airways disease. In addition, there was a chronic-looking small left apical pneumothorax, which on review of serial chest radiographs had been present for several months. There was marked thickening of the visceral pleura and widespread pleuroparenchymal tags in the upper zones.

He eventually died at the age of 23 years because of progressive respiratory failure. Postmortem histological analysis revealed extensive obliterative bronchiolitis and subpleural and paraseptal fibroelastosis (Figures 2a and b).

\section{Case 3}

A 16-year-old male underwent a sibling bone marrow transplantation for acute lymphoblastic leukaemia. Immediately after bone marrow transplantation, he developed respiratory problems with cough, sputum production and recurrent respiratory tract infections. Fifteen years after bone marrow transplantation, bilateral pneumothoraces were diagnosed, which recurred in the following year and were treated with pleurodesis, at which time a video-assisted thoracoscopic bullectomy was also performed. 

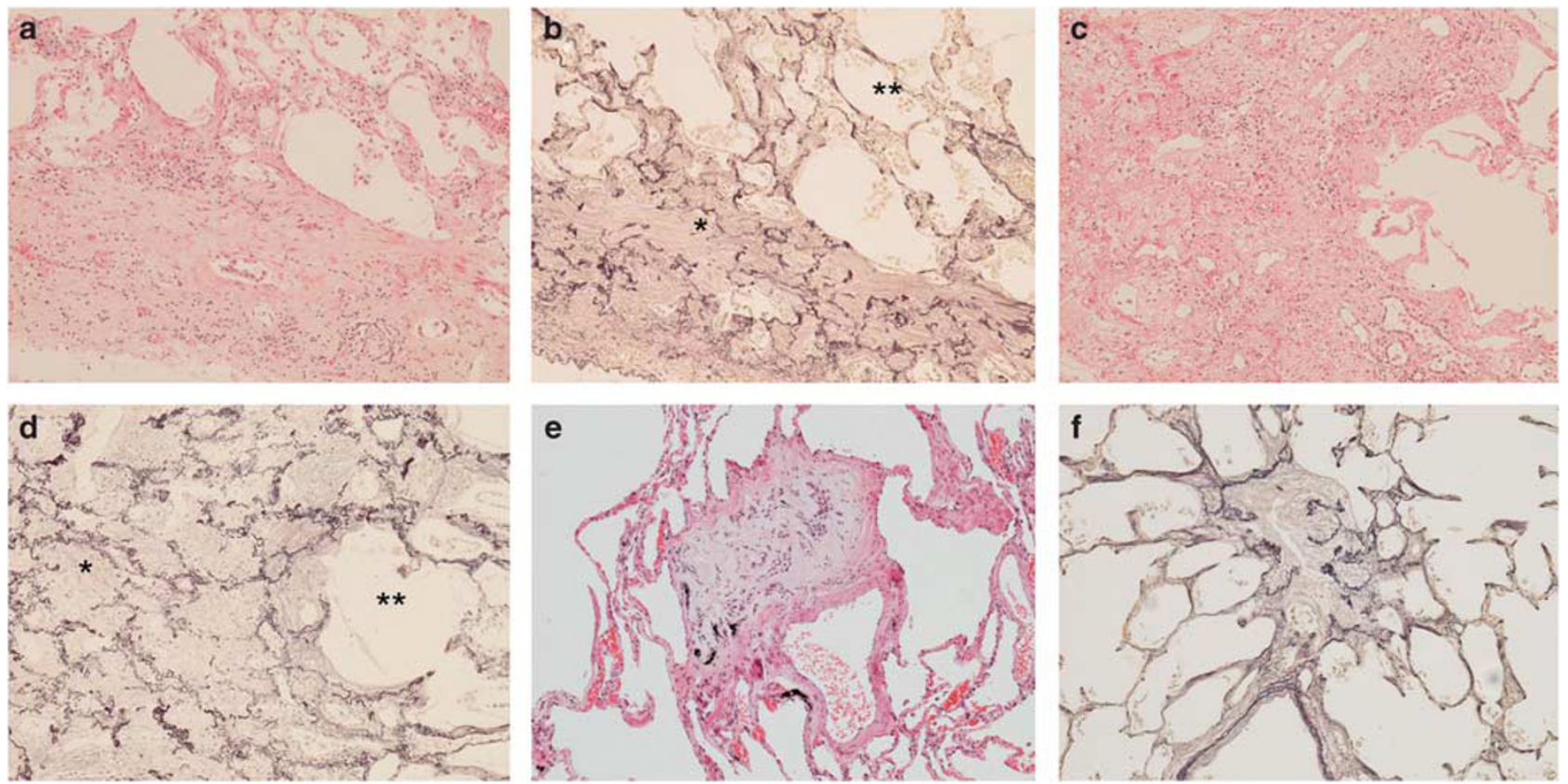

Figure 2 Histopathological spectrum of chronic pleuroparenchymal disease in bone marrow transplantation patients. (a and b) Subpleural fibroelastosis in case 2, showing intra-alveolar fibrosis $\left(^{*}\right)$ and adjacent unaffected lung parenchyma (**) (visceral pleural surface bottom left; Haematoxylin \& Eosin and Elastica-van Gieson stains, respectively). (c and d). Paraseptal parenchymal fibroelastosis in case 4 , again showing intra-alveolar fibrosis $(*)$ and adjacent unaffected lung parenchyma (**) (Haematoxylin \& Eosin and Elastica-van Gieson stains, respectively). Obliterative bronchiolitis with total obliteration of terminal bronchiole (e) and fibroblastic plugging of 'alveolar duct' $\left(\mathbf{f},{ }^{*}\right)$ in case 3.

High-resolution CT showed a moderate-sized right pneumothorax and a small left pneumothorax. There was a widespread mosaic attenuation pattern consistent with small airways disease. Several of the subsegmental bronchi were mildly dilated and slightly thick-walled. There was subpleural bleb formation and non-uniform thickening of the visceral pleura (Figure 1c).

The patient underwent pleural abrasion following video-assisted thoracoscopic biopsy of the leading edge of the right upper lobe, which revealed subpleural fibroelastosis, features consistent with pneumothorax, and obliterative bronchiolitis (Figures 2e and f). Half a year after the procedure, the patient was stable and well controlled on inhaled medication (ipratropium, salbutamol, beclomethasone), after which he was lost to follow-up.

\section{Case 4}

This 55-year-old woman underwent an autologous bone marrow transplant for the treatment of acute myeloid leukaemia. The transplant was uncomplicated, but she developed progressive interstitial pneumonitis with fibrosis. She was treated with steroids with some response to treatment, but was admitted with an acute exacerbation of her pulmonary disease 4 years and 4 months after bone marrow transplantation. Of note were IgM positivity for cytomegalovirus and evidence of herpes simplex virus on bronchoalveolar lavage. She was then treated with antibiotics and acyclovir, but her respiratory function continued to deteriorate and she died shortly afterwards.

High-resolution CT demonstrated a perihilar reticular pattern and pleuroparenchymal irregularity consistent with fibrosis. There was widespread ground-glass opacification within which traction of the airways was seen, denoting fine interstitial fibrosis. A small left pneumothorax was noted.

Postmortem examination revealed bilateral pleural effusions and relatively small, fibrotic lungs. Microscopically, sections from both lungs showed widespread pulmonary fibrosis with pleuroparenchymal fibroelastosis (Figures 2c and d) and focal obliterative bronchiolitis. Superimposed on these chronic changes there was widespread diffuse alveolar damage with abundant hyaline membrane formation, consistent with the ground-glass change seen on CT.

\section{Discussion}

This study presents four cases with pleuroparenchymal fibroelastosis associated with bone marrow transplantation. All patients presented with recurrent pneumothoraces and histological evidence of obliterative bronchiolitis; neither of which were 
a feature of those with idiopathic presentation of pleuroparenchymal fibroelastosis. Spontaneous pneumothoraces and obliterative bronchiolitis are now recognised as late complications of bone marrow transplantation and may actually be related to each other, possible through a peripheral check valve mechanism, as suggested in our earlier study. ${ }^{11}$ CTs in that study showed features similar to those currently described with small chronic pneumothoraces, upper zone subpleural fibrotic changes and diffuse abnormalities consistent with obliterative bronchiolitis, and the subpleural fibrosis was attributed to recurrent rupturing and healing of bullae secondary to obliterative bronchiolitis. The present review of the pathology suggests that the pleuroparenchymal fibroelastosis, with its progressive subpleural fibrosis and extensive elastin deposition, is as likely to be the cause of the pneumothorax rather than the effect, in the same way as lesser and more localised cicatricial subpleural changes secondary to hypoxia are thought to be the driving factors for spontaneous pneumothoraces. While it may be difficult to conceive how an area of increased subpleural fibrosis could be more prone to pneumothorax development (as intuitively, this could be viewed as leading to focally increased structural integrity), it should be borne in mind that the fibrosis in pleuroparenchymal fibroelastosis (especially in its earlier stages) is not continuous. Thus, lines of directly adjacent stiff fibrotic tissue and pliable native lung parenchyma exist, along which considerable tension is likely to occur throughout the ventilatory cycle, leading to pleural tearing and pneumothorax. Although speculative, this appears to be corroborated by previous studies showing an increased risk of pneumothorax in idiopathic pulmonary fibrosis (in which discontinuous subpleural fibrosis is also common), ${ }^{14}$ and higher risk of recurrent pneumothorax in pulmonary fibrosis. ${ }^{15}$

Histomorphologically, the so-called 'apical cap', which is a localised lesion of subpleural fibrosis that predominantly occurs in the apices of the upper lobes, enters the differential diagnosis of pleuroparenchymal fibroelastosis. ${ }^{16}$ However, several criteria can be used in combination to prise these two entities apart. First, apical caps tend to occur in older cigarette smokers (average age: 65.6 years, age range: 51 to 91 years), while pleuroparenchymal fibroelastosis also affects younger, non-smoking patients. Second, apical caps (as the name suggests) tend to be localised mass lesions in the upper lobe apices, and as such, they are often resected for a presumed diagnosis of carcinoma. On the other hand, pleuroparenchymal fibroelastosis, while displaying an upper lobe predominance, has a more diffuse subpleural distribution, which can also affect basal portions and other lobes. Third, histologically, apical caps are often associated with sclerotic pleural plaques and extensive alveolar collapse, which are not typically present in pleuroparenchymal fibroelastosis.
The aetiology of this specific pleuroparenchymal pattern of fibrosis in bone marrow transplantation patients remains elusive, although possible causes may include drugs (eg, induction or chemotherapy regimens), radiation-induced effects or graft-vs-host disease. Inflammation does not appear to be a dominant feature at the time of biopsy; however, an inflammatory component in earlier stages of the disease cannot be excluded. In the context of bone marrow transplantation, pleuroparenchymal fibroelastosis could be related to, but should be differentiated from, acute graft-vs-host disease-related changes. Thus, graft-vs-host disease may manifest itself as idiopathic pneumonia syndrome, which has been described to occur with an incidence of $\sim 10 \%$ following allogeneic bone marrow transplantation, ${ }^{1}$ with diffuse pulmonary infiltrates reflecting histopathological appearances of diffuse alveolar damage, organising or acute pneumonia and interstitial lymphocytosis. Idiopathic pneumonia syndrome completely resolves in a minority of patients and has a mortality rate of $\sim 74 \% .{ }^{17,18}$ It is frequently complicated by infections, pneumothorax, pneumomediastinum, subcutaneous emphysema, autoimmune polyserositis and pulmonary fibrosis. ${ }^{1,19}$ Idiopathic pneumonia syndrome tends to occur early after transplant (in the range of 1 to several weeks ${ }^{20}$ ), as opposed to pleuroparenchymal fibroelastosis, which appears to be of relatively late onset in our patient group. With respect to lung function, idiopathic pneumonia syndrome has the characteristics of acute widespread alveolar damage with decreased diffusion capacity and obstructive changes (as opposed to the restrictive ventilatory defect of pleuroparenchymal fibroelastosis), while CT imaging of this entity shows diffuse, multilobar infiltrates compared with the preferentially subpleural and apical location of pleuroparenchymal fibroelastosis. ${ }^{11,21}$ The positive imaging findings in (graft-vs-host disease-related) organising pneumonia and obliterative bronchiolitis also tend to have a more central distribution than pleuroparenchymal fibroelastosis. However, it is conceivable that the fibroelastosis seen in our patients could be a late complication of graft-vs-host disease, or specifically idiopathic pneumonia syndrome. The correlation of idiopathic pneumonia syndrome with serositis, fibrosis and pneumothorax may be of particular interest in our cases, as the latter two are certainly present and chronic serositis may have preceded the extensive pleuroparenchymal changes seen. Pleuritis, in the context of systemic lupus erythematosus, is known to have the potential to lead to pleural fibrosis in a minority of patients, ${ }^{22}$ as can drug-induced and rheumatoid pleuritis. ${ }^{23}$ Given organising pneumonia is a known complication of bone marrow transplantation, we hypothesise that pleuroparenchymal fibroelastosis may also represent persistence rather than resolution of intra-alveolar organisation. And although it is certainly conceivable that graft-vs-host diseaserelated, obliterative bronchiolitis could lead to a 
degree of subpleural atelectasis and collapse fibrosis, the pattern of fibrosis seen in FFPE is characterised more by alveolar filling with collagenous fibrosis, with retention and accentuation of alveolar septal elastin. Interestingly, a similar pattern of upper lobe fibrosis, distinct from apical caps, has previously been described as an idiopathic phenomenon in the Japanese literature, in which it has been termed as 'idiopathic pulmonary upper lobe fibrosis' or as Amitani disease. ${ }^{24-26}$

The range of symptoms and progression of disease can vary between pleuroparenchymal fibroelastosis patients. Although in some, the presentation is essentially benign and one of spontaneous pneumothorax, in others symptoms of dyspnoea and/or chronic cough predominate, and in yet others pleuroparenchymal fibroelastosis constitutes a disabling progression towards respiratory failure and potential death. Whereas in bone marrow transplantation, patients with an obstructive ventilatory defect due to obliterative bronchiolitis may be at the forefront; restrictive changes can certainly also occur concomitantly or in isolation, and these are likely to be related to pleuroparenchymal fibroelastosis. ${ }^{11}$

It is interesting to note that one of the patients in our current series is of paediatric age, as both graft-vs-host disease and pneumothorax following bone marrow transplantation in this age group tend to be less common than in the adult population, even in long-term follow-up (Dr Catherine Owens (Radiology Department, Great Ormond Street Hospital, London), personal communication).

We appreciate the limitations of the small size of our series, and additional studies are necessary to evaluate the relevance of the observed pattern of fibroelastosis in bone marrow transplantation patients. Expansion of this retrospective series could serve to elucidate underlying mechanisms and clinical risk factors for the development of fibrosis. Furthermore, pre-bone marrow transplantation CT scans are not available, and radiological confirmation of the absence of pulmonary changes before bone marrow transplantation is, therefore, not obtainable in this series. Clinically, however, respiratory symptoms did not manifest until several years after bone marrow transplantation and significant fibrotic changes are, therefore, unlikely to have been present at the time of transplantation.

Additional prospective studies will be required to determine the value of radiological and/or histopathological evidence of a pattern of pleuroparenchymal fibrosis as a prognostic marker for the development of recurrent/chronic pneumothoraces and of the likelihood of progressive pulmonary fibrosis in these patients.

\section{Disclosure/conflict of interest}

The authors declare no conflict of interest.

\section{References}

1 Afessa B, Peters SG. Noninfectious pneumonitis after blood and marrow transplant. Curr Opin Oncol 2008;20:227-233.

2 Afessa B, Peters SG. Chronic lung disease after hematopoietic stem cell transplantation. Clin Chest Med 2005;26:571-586.

3 Roychowdhury M, Pambuccian SE, Aslan DL, et al. Pulmonary complications after bone marrow transplantation: an autopsy study from a large transplantation center. Arch Pathol Lab Med 2005;129:366-371.

4 Sharma S, Nadrous HF, Peters SG, et al. Pulmonary complications in adult blood and marrow transplant recipients: autopsy findings. Chest 2005;128:1385-1392.

5 Chan CK, Hyland RH, Hutcheon MA. Pulmonary complications following bone marrow transplantation. Clin Chest Med 1990;11:323-332.

6 Khurshid I, Anderson LC. Non-infectious pulmonary complications after bone marrow transplantation. Postgrad Med J 2002;78:257-262.

7 Yousem SA. The histological spectrum of pulmonary graft-versus-host disease in bone marrow transplant recipients. Hum Pathol 1995;26:668-675.

8 Movsas B, Raffin TA, Epstein AH, et al. Pulmonary radiation injury. Chest 1997;111:1061-1076.

9 Worthy SA, Flint JD, Müller NL. Pulmonary complications after bone marrow transplantation: high-resolution CT and pathologic findings. Radiographics 1997;17:1359-1371.

10 Paz HL, Crilley P, Patchefsky A, et al. Bronchiolitis obliterans after autologous bone marrow transplantation. Chest 1992;101:775-778.

11 Sverzellati N, Zompatori M, Poletti V, et al. Small chronic pneumothoraces and pulmonary parenchymal abnormalities after bone marrow transplantation. J Thorac Imaging 2007;22:230-234.

12 Frankel SK, Cool CD, Lynch DA, et al. Idiopathic pleuroparenchymal fibroelastosis: description of a novel clinicopathologic entity. Chest 2004;126:2007-2013.

13 Becker CD, Gil J, Padilla ML. Idiopathic pleuroparenchymal fibroelastosis: an unrecognized or misdiagnosed entity? Mod Pathol 2008;21:784-787.

14 Iwasawa T, Ogura T, Takahashi H, et al. Pneumothorax and idiopathic pulmonary fibrosis. Jpn J Radiol 2010;28:672-679.

15 Lippert HL, Lund O, Blegvad S, et al. Independent risk factors for cumulative recurrence rate after first spontaneous pneumothorax. Eur Respir J 1991;4: 324-331.

16 Yousem SA. Pulmonary apical cap: a distinctive but poorly recognized lesion in pulmonary surgical pathology. Am J Surg Pathol 2001;25:679-683.

17 Kantrow SP, Hackman RC, Boeckh M, et al. Idiopathic pneumonia syndrome: changing spectrum of lung injury after marrow transplantation. Transplantation 1997;63:1079-1086.

18 Crawford SW, Longton G, Storb R. Acute graft-versus host disease and the risks for idiopathic pneumonia after marrow transplantation for severe aplastic anemia. Bone Marrow Transplant 1993;12:225-231.

19 Afessa B, Litzow MR, Tefferi A. Bronchiolitis obliterans and other late onset non-infectious pulmonary complications in hematopoietic stem cell transplantation. Bone Marrow Transplant 2001;28:425-434.

20 Keates-Baleeiro J, Moore P, Koyama T, et al. Incidence and outcome of idiopathic pneumonia syndrome in 
pediatric stem cell transplant recipients. Bone Marrow Transplant 2006;38:285-289.

21 Afessa B, Peters SG. Major complications following hematopoietic stem cell transplantation. Semin Respir Crit Care Med 2006;27:297-309.

22 Man BL, Mok CC. Serositis related to systemic lupus erythematosus: prevalence and outcome. Lupus 2005;14:822-826.

23 Huggins JT, Sahn SA. Causes and management of pleural fibrosis. Respirology 2004;9:441-447.
24 Morimoto A, Mochizuki Y, Nakahara Y, et al. [Case of idiopathic pulmonary upper lobe fibrosis]. Nihon Kokyuki Gakkai Zasshi 2010;48:944-949.

25 Kobayashi Y, Sakurai M, Kushiya M, et al. [Idiopathic pulmonary fibrosis of the upper lobe: a case report]. Nihon Kokyuki Gakkai Zasshi 1999; 37:812-816.

26 Shiota S, Shimizu K, Suzuki M, et al. [Seven cases of marked pulmonary fibrosis in the upper lobe]. Nihon Kokyuki Gakkai Zasshi 1999;37:87-96. 